\title{
Holographic Capture and Display Systems in Circular Configurations
}

\author{
Tomasz Kozacki, Małgorzata Kujawińska, Grzegorz Finke, Weronika Zaperty, and Bryan Hennelly
}

\begin{abstract}
This paper presents a novel method of multi-spatial light modulator (SLM) holographic image display that enables wide angle reconstruction of images of real world objects. The image data are delivered by means of digital holography. The capture and display systems are arranged in a circular configuration. In order to support the proper information flow between the multi-sensor capture and multi-SLM display systems we perform analysis using the Wigner distribution function. We also consider a mismatch of the capture and display configurations as well as the visual perception of the displayed image. Experimental results based on the reconstruction of real world scenes are presented that demonstrate the validity of the theoretical solutions. A single camera is used to record the digital holograms, where the object is rotated between captures, and these holograms are then displayed on a circular configuration of SLMs. The results show the limitation of multi-SLM holographic displays in terms of visual perception. These problems arise from the limited angular distribution of the SLMs on a display circle and the presence of gaps between the SLMs.
\end{abstract}

Index Terms-Digital holography, holographic displays, 3D displays, Wigner distribution, spatial-light modulation (SLM).

\section{INTRODUCTION}

$\mathbf{I}$ N RECENT HISTORY of visual multimedia, there has been a rapid development of three-dimensional data content capture and display. The main areas of development include stereoscopic and autostereoscopic systems, head mounted displays, integral photography and digital holography [1], [2]. Most of the systems are based on a certain manipulation of two or more 2D images which, when converted by our brain provide the depth information which is very important for immersive and presence feeling [3]. However, the most advanced imaging is represented by so called "True-3D" which is realized by the physical duplication of a light distribution in a volume of interest. Holography is a sophisticated true 3-D method in which the full object wavefield information can be captured and later optically

Manuscript received June 23, 2011; revised August 25, 2011; accepted August 29, 2011. Date of current version March 28, 2012. The research leading to these results has received funding from the EU 7th Framework Programme FP7/2007-2013 under Agreement 216105 ("Real 3D” Project) and the statutory funds of Warsaw University of Technology.

T. Kozacki, M. Kujawinska, G. Finke, and W. Zaperty are with the Photonics Department, Warsaw University of Technology, 02-525 Warsaw, Poland (e-mail: t.kozacki@mchtr.pw.edu.pl,m.kujawinska@mchtr.pw.edu.pl, g.finke@mchtr.pw.edu.pl,w.zaperty@mchtr.pw.edu.pl).

B. Hennelly is with the Department of Computer Science, National University of Ireland Maynooth, County Kildare, Ireland (e-mail: bryanh@cs.nuim.ie).

Color versions of one or more of the figures are available online at http:// ieeexplore.ieee.org.

Digital Object Identifier 10.1109/JDT.2011.2167955 reconstructed. Holographic imaging was pioneered by Leith and Upatnieks in 1962 [4] using materials. However due to several limitations connected with the digital data capture (limited space bandwidth product of sensors and the limited bandwidth of hologram transmission systems) the implementation of 3-D holographic displays had not been realized. This area of research remained dormant until 1990 when high resolution cameras and spatial-light modulators (SLMs) became available and the concept of digital holography was introduced into multimedia [5].

Due to limited resolution and aperture, a single SLM has a limited angular view and this is the main problem affecting the user experience of observing optically reconstructed holographic images. To overcome this problem several attempts have been made to enhance this feature by employing innovative optical holographic display modules with several tilled SLMs [6]-[8]. There are two major display approaches, basing on flat geometry [9] and a circular one [10], [11]. In proposed display in a paper we utilize a circular configuration. For the purpose of extending the viewing angle this configuration is the preferable one. In this circular configuration we not increase the resolution of reconstructed image because the wavefield from the SLMs do not coherently interfere with each other as this would require a very careful and accurate measurement of the CCD positions and placement of the SLMs (to a fraction of a wavelength). However, we do extend the viewing angle. In the planar display configuration the maximum viewing angle is a function of the display pitch and extension of the number pixels does not allow us to increase the viewing angle over the maximum one. Earlier work using SLMs focused on holographic imaging of computer generated holograms or stereograms which have no restrictions or limitations connected with a data capture system. A big challenge is still to provide an efficient digital holographic approach for the capture and display of real world 3D objects. The concept of such a system with multiple cameras in a circular configuration around a 3D scene and the respective multiple SLM display is presented in Fig. 1 and was proposed recently in [12] and [13].

However, in order to put this concept into full operation in the paper we address several theoretical, as well as practical, questions including the description of the information content flow from a multi-CCDs capture to a multi-SLMs display configurations (Sections II and III). Particularly, we discuss problems of: limited angular size of the capture and display systems, the existence of gaps between the CCDs and SLMs in a circular arrangement, mismatch of capture and display parameters (wavelength, pitch size) and visual perception. For the analysis of the above-mentioned problems we have applied the Wigner distribution function, (WDF) [14], [15], which provides 


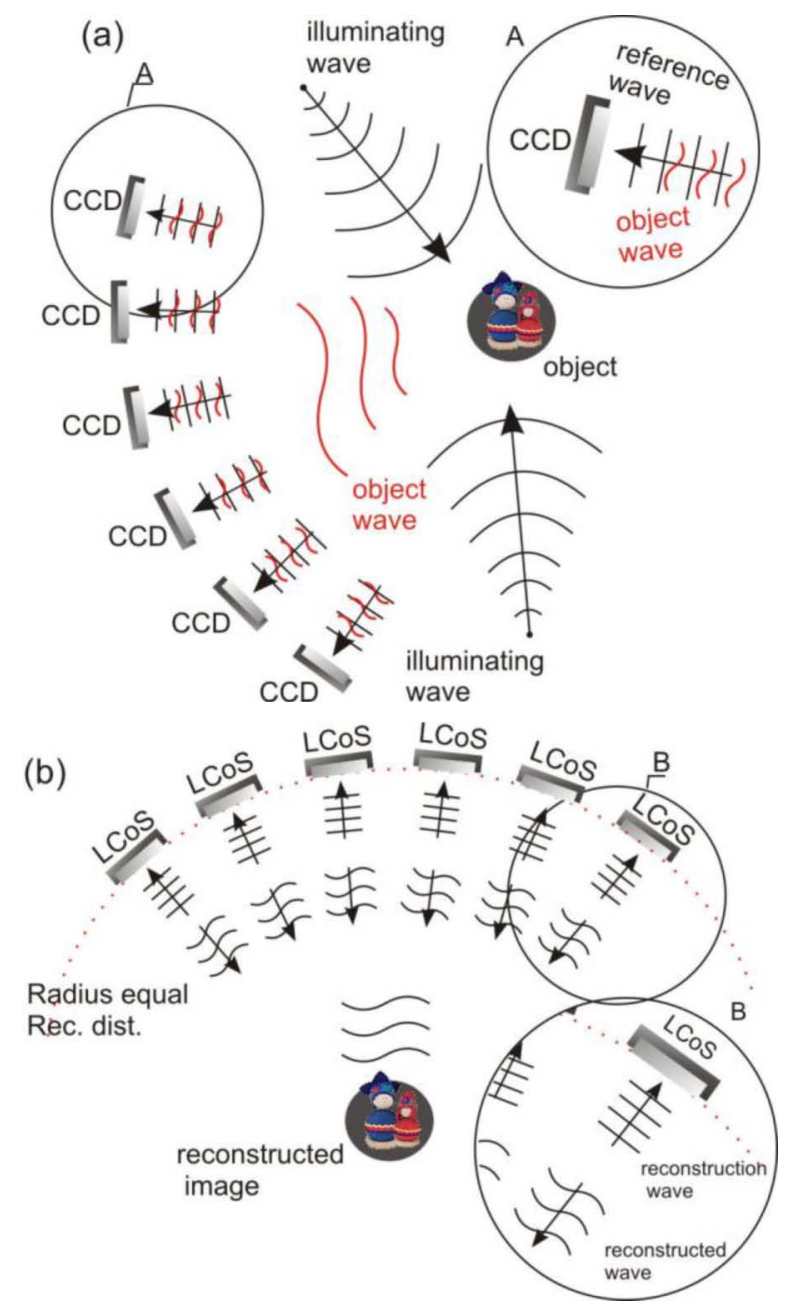

Fig. 1. Schemes of (a) holographic data capture and (b) display by multiple CCDs and LCoS SLMs in the circular configuration.

the joint space-spatial frequency representation of real and complex signal. In this work we utilize Wigner charts for the analysis of the multi-sensor and multi-SLM systems.

It is important to note, that for our experimental results, we do not use a multi-camera configuration. Instead, we use a single camera system with a static object located at a rotary table (Section IV-A). This gives us maximum flexibility in choosing our camera "positions" to correspond to our multi-SLM configuration. The experiment presented in Sections IV-C and IV-D utilizes real-world objects holographic data as well as computer generated holograms in order to clearly prove our theoretical consideration. We present experiments showing reconstructions of different real world scenes. The scene sizes are chosen to correspond to the field of view of the display.

\section{THEORY OF Wide ANGLE HOLOGRAPHIC CAPTURE}

In order to gain insight into the general capture setup shown in Fig. 1(a) we employ phase space diagrams or "Wigner charts." This plan view representation of the WDF allows us to derive first order approximations to some important properties of our multi-camera configuration. We will apply our analysis to only two adjacent cameras and in this case we can state, that these two cameras are located in the same plane at a distance $z_{1}$ from
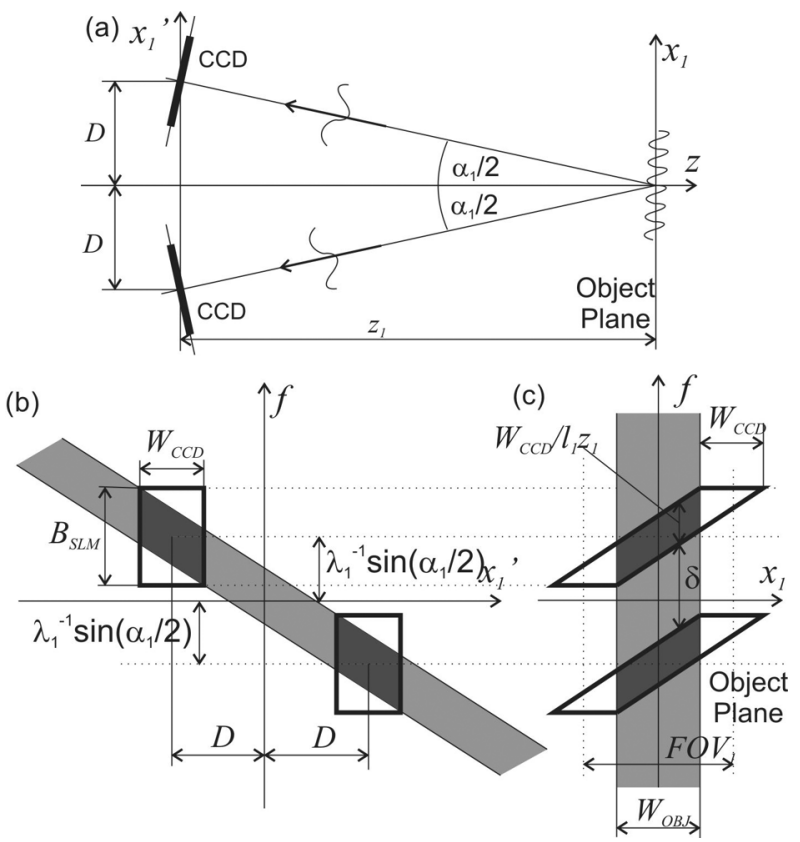

Fig. 2. Multi-CCDs capture setup: (a) configuration for capturing two portions of the object wavefield; (b) WDF of the signal in the capture plane; and (c) corresponding WDF mapped back to the original object plane.

the object and they are separated by an angle $\alpha_{1}$. We assume that the paraxial approximation can be used to describe propagation from the object plane to the plane of the two cameras. Under this approximation, the WDF is mapped as follows:

$$
W(x, f) \rightarrow W(x-\lambda z f, f) .
$$

In Fig. 2(a) we show an illustration of the two-camera capture setup. The two cameras are positioned in a plane at a distance $z_{1}$ from the object plane. The cameras are located at a distance $D$ either side of the optical axis and the angles they subtend to the optical axis are $\alpha_{1} / 2$ and $-\alpha_{1} / 2$. These two cameras are assumed to be identical with a width given by $W_{\mathrm{CCD}}$ and a bandwidth given by $B_{\mathrm{CCD}}=1 / \Delta_{1}$, where $\Delta_{1}$ is the pixel pitch of the camera.

The WDF of the object signal is shown for the object plane in Fig. 2(c) and for the capture plane in Fig. 2(b). In both cases the lighter shaded areas represent this full continuous signal. The object will have a certain width $W_{\text {OBJ }}$ and this explains the finite support of the WDF shown in Fig. 2(c). The bandwidth of the signal can extend over infinity and hence the signals are shown not to be bounded in the $f$ axis. In Fig. 2(b) we illustrate the WDF of the propagated signal. The horizontal sharing results in the signal having a finite local bandwidth and hence a $\mathrm{CCD}$ can be used to capture the complex wave field using interferometry. The WDFs of the two cameras in the capture plane are shown in Fig. 2(b) using thick black lines. These rectangular WDFs have a width and bandwidth given by $W_{\mathrm{CCD}}$ and $B_{\mathrm{CCD}}$. Their centers are located at $\pm D$ in the $x$ direction and $\pm \lambda_{1}^{-1} \sin \left(\alpha_{1} / 2\right)$. These two CCDs capture a portion of the energy of the object signal. This energy is shown as the darker shaded region in the figure.

In Fig. 2(c) we show the corresponding region of energy in the original object plane. We have presented the WDF of the 
CCDs in this plane as well. It can be shown using basic geometry that the local bandwidth of the regions is given by $W_{\mathrm{CCD}} / \lambda_{1} z_{1}$. Therefore the gap between the two shaded regions in Fig. 2(c) is given by

$$
\delta_{1}=2 \frac{\sin \left(\alpha_{1} / 2\right)}{\lambda_{1}}-\frac{W_{\mathrm{CCD}}}{\lambda_{1} z_{1}} .
$$

The equation gives an estimate of the frequency gap between two adjacent cameras. The same analysis can be applied to any two adjacent cameras in a wider assembly. In order to capture the full continuous field we require $\delta_{1}$ to be zero. From (1) above we can see that this will be achieved if

$$
\sin \left(\alpha_{1} / 2\right)=\frac{W_{\mathrm{CCD}}}{2 z_{1}} .
$$

This condition would clearly ensure that the active camera faces were just touching. If this condition is fulfilled the capture fill factor equals unity $\left(\mathrm{FF}_{1}=1\right)$. This is not possible in general due to the external components of the camera. In Fig. 2(c) we also present the total field of view, $\mathrm{FOV}_{1}$ that can be reconstructed by the captured digital holograms without aliasing. It can be shown that this is given by

$$
\mathrm{FOV}_{1}=\lambda z_{1} B_{\mathrm{CCD}}=\frac{\lambda z_{1}}{\Delta_{1}}
$$

\section{Theory OF Wide ANGLE Holographic Display}

In order to reconstruct and display the captured wide angle complex image a holographic display consisting of multiple spatial light modulators has to be applied [see Fig. 1(b)]. In addition we have to consider the physical dimensions of the applied SLMs (the display fill factor $\mathrm{FF}_{2}$ ). Moreover the capture and display devices are usually characterized by different sampling parameters and the capture and reconstruction wavelengths can differ as well. Below we will discuss the influence of the mismatch of capture and display systems on the reconstructed image.

\section{A. Mismatch in Sampling and Wavelength}

The captured optical field given by discrete samples is to be replicated by spatial light modulators and using a different wavelength $\lambda_{2}$. A single SLM is characterized by the sampling parameters: a number of pixels $N_{2}$ and a size of a pixel $\Delta_{2}$. Let us assume that the experiential capture-display devices CCDs and SLMs have comparable numerical space bandwidth products (number of pixels). Therefore we can directly modulate the SLM with the discrete captured field. Applying such a strategy we display holographic data with transverse magnification:

$$
M_{t}=\Delta_{2} / \Delta_{1}
$$

and longitudinal (axial) magnification $M_{l}$, where $z_{2}=z_{1} M_{l}$ :

$$
M_{l}=\frac{\lambda_{1}}{\lambda_{2}} M_{t}^{2}=\frac{\lambda_{1} \Delta_{2}^{2}}{\lambda_{2} \Delta_{1}^{2}} .
$$

Subscripts $_{1} \operatorname{and}_{2}$ relate to the capture and reconstruction processes, respectively. The relations between magnifications given by (4) and (5) is well known in the field of classical holography and diffractive optics [16]. Diffractive optical elements
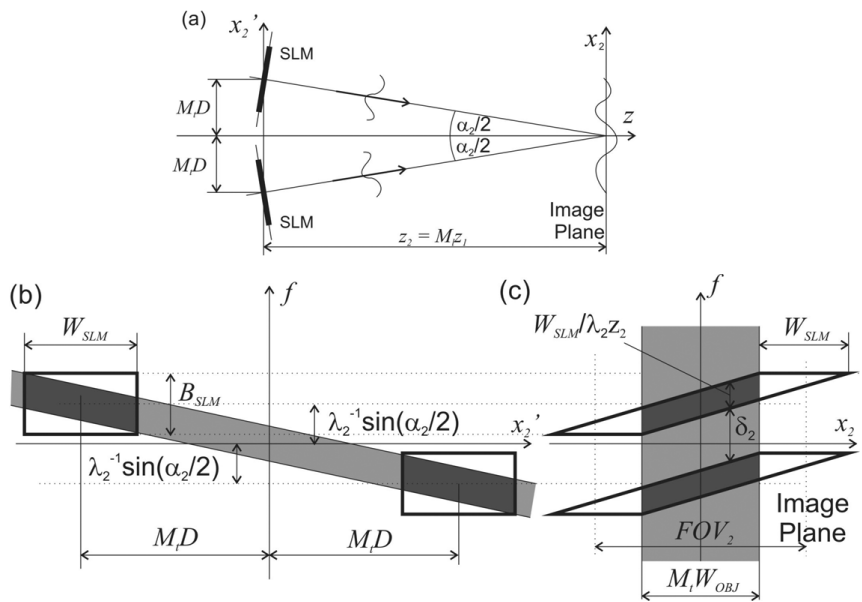

Fig. 3. Multi-SLMs display setup: (a) configuration for two SLMs; (b) WDF of the signals in the SLM plane; and (c) corresponding WDFs in the reconstruction plane.

suffer from strong chromatic aberration. When linking the capture and display systems, there is another important magnification to consider, i.e., angular magnification, $M_{a}$. It can be derived that the angular magnification takes the form

$$
M_{a}=\frac{\sin \left(\alpha_{2} / 2\right)}{\sin \left(\alpha_{1} / 2\right)}=\frac{M_{t} z_{1}}{z_{2}}=\frac{M_{t}}{M_{l}}=\frac{\lambda_{2}}{\lambda_{1} M_{t}} .
$$

Concluding our previous discussion, we state that the capturedisplay system mismatch gives the following.

- Ttransversely magnified $\left(M_{t}\right)$ hologram will result in a transversely magnified $\left(M_{t}\right)$ image (also $M_{t}$ ).

- Reconstruction distance and 3D depth are increased by a factor of the longitudinal magnification $\left(M_{l}\right)$.

- Angular separation of SLMs is increased by a factor of the angular magnification $M_{a}$ (when compared with the angular separation of CCDs).

\section{B. Configuration Mismatch}

We use the WDF to derive some first order approximations to selected important properties of our display configuration. Once again we apply our analysis to only two adjacent elements and in this case we can state that these two SLMs are located in the same plane at a distance $z_{2}$ from the object and they are separated by an angle $\alpha_{2}$. We again assume that the paraxial approximation can be used to describe propagation from the SLM plane to the image plane.

The SLMs in the holographic display are directly modulated with the captured phases [17]. The first implication of having the magnification factor $M_{t}$ associated with the size mismatch is that the SLMs must be placed at locations $\pm M_{t} D$ in order to simulate a uniformly magnified hologram. This is illustrated in Fig. 3(a). The second implication is that the reconstructed image appears in focus at a distance $z_{2}$ away from the SLM plane where $z_{2}=M_{l} z_{1}$, and $M_{l}$ is defined by (5) above. This results in an angular separation between the SLMs, $\alpha_{2}$, which is defined by using (6) above.

In Fig. 3(a) we show that the angles the SLMs subtend to the optical axis are $\alpha_{2} / 2$ and $-\alpha_{2} / 2$. The two SLMs are assumed to be identical with a width given by $W_{\mathrm{SLM}}$ and a bandwidth given 
by $B_{\mathrm{SLM}}=1 / \Delta_{2}$. From earlier discussions we can assume that $W_{\mathrm{SLM}}=M_{t} W_{\mathrm{CCD}}$ and $B_{\mathrm{SLM}}=B_{\mathrm{CCD}} / M_{t}$. The space bandwidth products of the SLM and the camera are therefore equal and they share the same number of pixels. We also note that this implies the WDFs of the CCD and SLMs will have equal areas in the $x-f$ plane.

The WDF of the magnified (by $M_{t}$ ) object signal is shown for the SLM plane in Fig. 3(b). The result of magnification is to stretch the original WDF (shown in Fig. 2(b)) along the $x$ direction, resulting in a narrowing of the WDF in $f$ by the same factor. The WDFs of the two SLMS are shown in Fig. 3(b). Their centers are located at $\pm M_{t} D$ in the $x$ direction and $\pm \lambda_{2}^{-1} \sin \left(\alpha_{2} / 2\right)$. The energy of the signal that is displayed on the two SLMs is shown as the darker shaded regions in the figure. These regions are stretched (magnified) versions of the dark regions shown in Fig. 2(b).

In Fig. 3(c), we show the corresponding region of energy in the reconstructed image plane, at a distance $z_{2}$ away. We have also presented the WDF of the SLMs in this plane. Once again it can be shown using basic geometry that the local bandwidth of the regions is given by $W_{\mathrm{SLM}} / \lambda_{2} z_{2}$. Therefore the gap between the two shaded regions in Fig. 3(c) is given by

$$
\delta_{2}=2 \frac{\sin \left(\alpha_{2} / 2\right)}{\lambda_{2}}-\frac{W_{\mathrm{SLM}}}{\lambda_{2} z_{2}}=\frac{\delta_{1}}{M_{t}} .
$$

The equation gives an estimate of the frequency gap between two adjacent SLMs. The same analysis can be applied to any two adjacent SLMs in a wider assembly. In Fig. 3(c) we also show the field of view of the display, $\mathrm{FOV}_{2}$. This is the area of the display that is free from aliasing from neighboring orders and is given by

$$
\mathrm{FOV}_{2}=\lambda z_{2} B_{\mathrm{SLM}}=\frac{\lambda z_{2}}{\Delta_{2}}=M_{t} \mathrm{FOV}_{1} .
$$

It is also shown in the figure, that the object width has been scaled by the factor $M_{t}$. If we assume that in our capture system we can get a hologram for any object perspective, we impose display geometrical limitations only and then use these to define appropriate camera angles and positions. The SLM applied for holographic display is in our case the Holoeye 1080P liquid crystal on silicon (LCOS) SLM. The frame of the SLM display is $24 \mathrm{~mm}$ and it determines the distance between the active areas of neighboring SLMs. Since the active area of SLM is $15.36 \mathrm{~mm}$, the maximum display angular fill factor is $\mathrm{FF}=\approx$ $15.36 / 24=0.64$. We have purposefully removed subscript 2 form $\mathrm{FF}$ symbol, value of $\mathrm{FF}$ is equal for capture and display systems. We can determine $M_{t} D=12 \mathrm{~mm}$, half the width of the entire SLM. Assuming that $z_{2} \gg 12 \mathrm{~mm}$ we can approximate

$$
\sin \left(\alpha_{2} / 2\right) \approx \frac{M_{t} D}{z_{2}}=\frac{12[\mathrm{~mm}]}{z_{2}} .
$$

Using (6) we can deduce that this forces the minimum angular separation in the capture set up to be

$$
\alpha_{1}=2 \sin ^{-1}\left(\frac{12[\mathrm{~mm}]}{M_{a} z_{2}}\right) .
$$

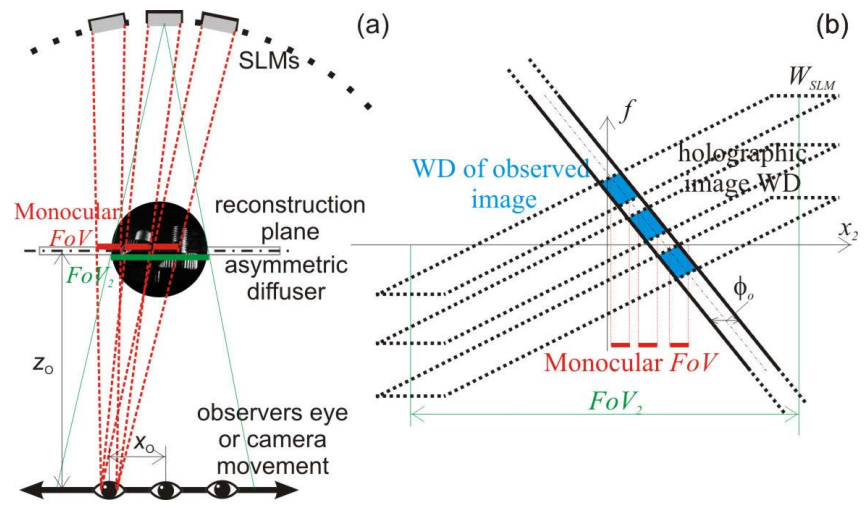

Fig. 4. Visual perception analysis of multi-SLM holographic display system (a) configuration (b) WD representation.

It should be mentioned that the optical field that is displayed is not a direct magnified version of the captured field since during the reconstruction process we disregard the object wave amplitude information. However, the image of a scattering object is well approximated by the phase distribution only at a plane distant from the object [18]. Having this in mind we modulate our display SLMs with phase only and this results in a good approximation of a complex object image.

\section{Viewing Reconstructed Holograms}

In this section, we focus our attention to multi-SLM holographic image observation. Once again the analysis is performed by means of WDF graphical representation of the holographic imaging process, this time we introduce an eye aperture characterized by a size $\left(\phi_{0}\right)$ and position $\left(x_{\mathrm{o}}\right)$. Study of visual perception of single SLM display is presented elsewhere [19].

In Fig. 4, the single eye off-axis observation of the real holographic image is illustrated using the Wigner chart. The eye position is arbitrary, so the addition of second eye is straight forward. Wigner charts of the eye and the holographic image are shown for the reconstruction plane. The Wigner chart of the reconstructed image is given by the three horizontally sheared signals (see Fig. 3(b) for a more clear illustration of this for the case of two SLMs). The field of view of the reconstruction plane is given by (8) above and is marked in the figures as $\mathrm{FoV}_{2}$. The Wigner chart representing the effect of the eye in the reconstructed image plane is found by back propagating the WDF of a rectangular function to the image plane. The overlap represents the energy from the image that is actually captured by the eye. This overlap allows us to determine the monocular FoV(MFoV). In the circular configuration the $\mathrm{FoV}_{2}$ can be approximated by a single SLM. Additional SLMs are added in order to extend angular perspective only. In Fig. 4, FoV 2 is represented with green color. We have illustrated the situation where the $\mathrm{FoV}_{2}$ is larger than the size of observed image (MFoV).

The FoV according to WDF chart is $B_{\mathrm{SLM}} \lambda z_{2}$. To obtain a value of $M F o V$ we assume first that there are no gaps in the 
system. In this situation we get a continuous $M F O V$. For $N$ SLMs we obtain

$$
\mathrm{MFoV}=\frac{\mathrm{NW}_{\mathrm{SLM}} z_{0}}{z_{o}+z_{2}}
$$

where $z_{o}$ is an observation distance. For an off axis eye observation, we get a MFoV shift in the image plane

$$
x_{\mathrm{MFoV}}=\frac{z_{2} x_{o}}{z_{o}+z_{2}} .
$$

We assume here that SLMs are distributed on circle symmetrically around the optical axis of the display. If we now consider the holographic display, where SLMs are distributed on circle with gaps $(\mathrm{FF} \neq 0)$ we get

$$
\mathrm{MFoV}_{\mathrm{FF}}=\operatorname{MFoV} \frac{(N-1+\mathrm{FF})}{\mathrm{FF} \cdot N} \text {. }
$$

This is MFoV for display with gaps characterized by fill factor. The size of viewed gap is simply

$$
\mathrm{MFoV}_{\text {gap }}=\mathrm{MFoV} \cdot N^{-1}\left(\mathrm{FF}^{-1}-1\right) .
$$

In deriving (13) and (14) we use criteria of 50\% loss of observed energy and resolution. This is presented in Fig. 4(b), where size of MFoV is computed from projection of central line of eye slit in WD on $\mathrm{x}$ axis. Therefore when we view the reconstruction, size of a gap appears to be smaller.

\section{Multi-SLM HolOgRAPHIC CAPTURE AND DISPLAY-EXPERIMENT}

\section{A. Holographic Capture System in Circular Configuration}

The simplified system, which in our experiments provides $360^{\circ}$ holographic capture for static 3D objects is presented in Fig. 5. A macroscopic object is placed at a rotational table and the holograms of different object angular views are captured sequentially by a single CCD camera. The fill factor of the angular data representing an object may be adjusted arbitrarily, so the full-wave field representing an object in space can be captured. The laser diode with $\lambda_{1}=785 \mathrm{~nm}$ is used as the coherent light source, while the camera parameters are: the number of pixels is $N_{1 x} \times N_{1 y}(1656 \times 1060)$ and the pitch is $\Delta_{1}=6.45 \mu \mathrm{m}$. The plane reference beam is directed at the camera by a mirror $\mathrm{M}$, which is attached to a piezoelectric transducer (PZT). The setup enables the capture of off-axis and in-line Fresnel holograms. Off-axis holograms can be captured by slightly rotating the second beamsplitter. In the case of in-line holograms the twin image and DC terms are removed by using a phase-shifting interferometic (PSI) technique [20] (i.e., by capturing at least three phase-shifted holograms, the phase shifts are introduced by translating the mirror in reference beam). The best usage of detector bandwidth is realized in the case of in-line holograms, so this is the preferred capture configuration.

\section{B. Multi-SLMs Display System}

A more detailed practical realization of the holographic display shown in Fig. 1(b) is presented in Fig. 6. The display is

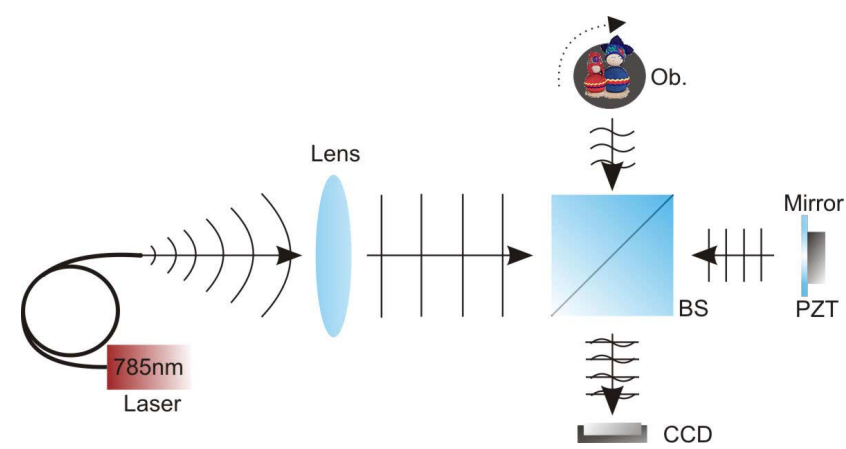

Fig. 5. Scheme of the DH capture system for static objects (Ob). (BS-beam splitter; PZT—phase shifter.)

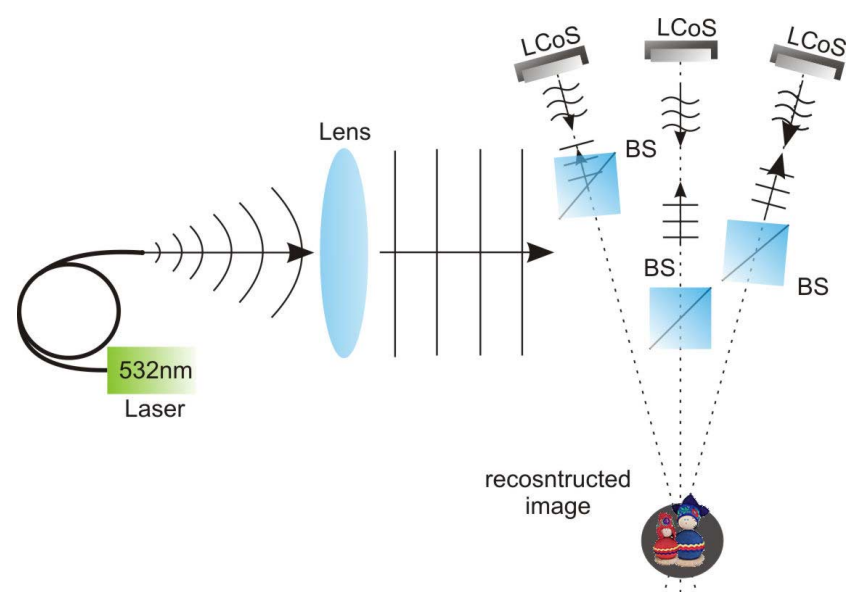

Fig. 6. Multi-LCoS SLM display system with normal illumination.

constructed so the captured field can be directly reproduced by SLMs. To reconstruct the holograms acquired in the rotational capture geometry a multiple plane wave illumination is required. During capture each CCD points toward a center point. Therefore every SLM must be illuminated by a plane wave with a wave vector normal to the SLM and each SLM must point towards the central rotation axis of the capture geometry. In the experimental setup this is realized by an accurate angular adjustment of beam splitting cubes and SLMs. The display is based on three phase only Holoeye LCOS SLMs (model HEO 1080P) with the parameters: $N_{2 x} \times N_{2 y}(1920 \times 1080)$ and $\Delta_{2}=8 \mu \mathrm{m}$. The light source is the Nd-YAG laser with $\lambda_{2}=532 \mathrm{~nm}$. The illumination beams are linearly polarized along the LC molecules and therefore only the phases of these beams are modulated. The display is configured with a fill factor of $F F=0.51$. This controls the value of the angular step of the capture system, which in turn determines the angles of perspectives.

\section{Computer Generated Holograms Reconstructions in Multi-SLMs Display}

In the multi-SLM display (see Fig. 6) we have reconstructed two types of holograms: digital holograms of real world objects and computer generated holograms of synthetic scenes. First we present the results of the reconstruction from three SLM display of synthetic holograms. The holograms are simulated for an object consisting of two sets of letters-1) REAL 3D and 2) BY WUT - the words are located in longitudinally separated planes 


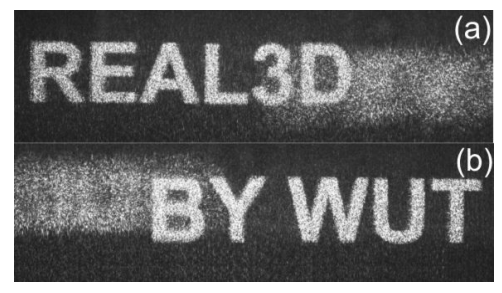

Fig. 7. Optical reconstructions of holographic scene for simulated digital holograms. (a) CCD captured real image at $670 \mathrm{~mm}$ and (b) and at $700 \mathrm{~mm}$.

by the distance $\Delta z_{2}=3 \mathrm{~mm}$. We present this experiment to demonstrate our method of calibrating the SLM positions. Each SLM gives a different perspective of the sets of letters. We adjust the angle of our SLMs and their positions so that the real images from the individual SLMs overlap in 3D reconstruction space. In Fig. 7(a) we show a real image captured at one reconstruction plane and in Fig. 7(b) at the second one.

\section{Digital Holograms Reconstructions in Multi-SLMs Display}

In our display we can reconstruct holograms of a real world object using multiple spatial light modulators. We believe this is the first time that such a display has been described in the literature. First we show the result of reconstructing a holographic scene of a set of screws. The multi-SLM display configuration extends the VFoV in the $x$ direction only. Therefore in Fig. 8 we present views of the optoelectronic reconstructions by two means: with an additional asymmetric diffuser [see Fig. 8(a)] or with an additional eyepiece [see Fig. 8(b)]. Without these elements we can view only part of the reconstruction in the $y$ direction only. While capturing images and videos of the reconstructed scenes we have taken special care to present them as they are viewed by a human eye i.e., the views are captured with a digital camera adjusted to human eye observation conditions. The entrance pupil diameter of digital camera is set to $8.2 \mathrm{~mm}$, the value is close to human observation condition in the dark room (eye aperture diameter $8 \mathrm{~mm}$ ). All photos are taken with the digital camera placed on a linear stage at a distance $z_{0}$ from the reconstruction plane and the digital camera is moved on a linear stage as presented in Fig. 4.

The digital holograms of the two screws were captured for the object position $z_{1}=278.8 \mathrm{~mm}$ and rotated through a series of angular steps $\Delta_{\alpha 1}=5^{\circ}$. Each SLM replays the phase of a digital hologram captured for different object perspective. The series of three holograms is captured using phase shifting technique so the object wavefront phase is accurately recovered. The capture and display systems are characterized by a mismatch in wavelength: $\lambda_{1}=785 \mathrm{~nm}, \lambda_{2}=532 \mathrm{~nm}$ and pixel pitch: $\Delta_{1}=6.45 \mu \mathrm{m}, \Delta_{2}=8 \mu \mathrm{m}$. The mismatch of the capture-display geometries results in the magnifications $M_{t}=1.2403$, $M_{l}=2.27$, and $M_{a}=0.5464$. This gives instantly: $\Delta_{\alpha 2}=$ $2.72^{\circ}$ and $z_{2}=633 \mathrm{~mm}$. In Fig. 8 we show the view of the real holographic image generated by the display. The angular field of view $(\mathrm{AFoV})$ of the real image can be computed from the image frequency span represented in Fig. 3(c)

$$
\mathrm{AFOV}=\sin ^{-1}\left(2 \sin \alpha_{2}+\frac{W_{\mathrm{SLM}}}{z_{2}}\right)=6.85^{\circ} .
$$

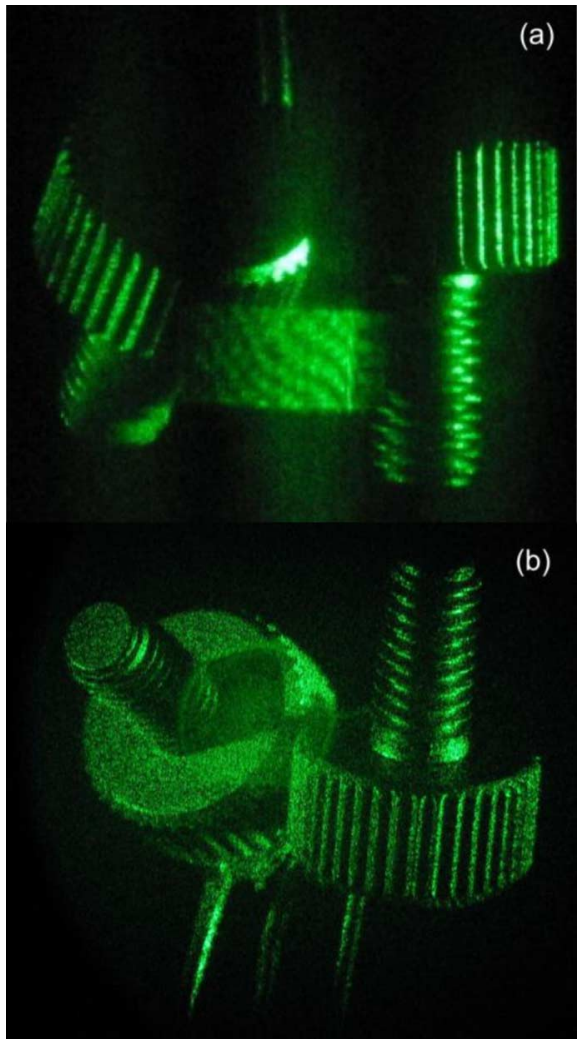

Fig. 8. Optical reconstructions of the holographic scene captured with digital holography. (a) Views captured with the asymmetric diffuser and digital camera, (Media 1). (b) Views captured with the eyepiece lens and digital camera (Media 2).

We mention here, that for exemplary reconstruction distance of $z_{2}=300 \mathrm{~mm}$, we have AFOV $=14.53^{\circ}$. However, then we have short capture distance $\left(z_{1}=132 \mathrm{~mm}\right)$ and small reconstructed images $\left(\mathrm{FOV}_{2}=20 \mathrm{~mm}\right)$.

The $\mathrm{MFoV}_{\mathrm{FF}}$ according to (13) is $33.4 \mathrm{~mm}$. The reconstructed object has size $33 \mathrm{~mm}$ in $x$ direction. Both values are of the same magnitude and smaller than $\mathrm{FoV}=42.08 \mathrm{~mm}$. The captured scene and observation distance $\left(z_{o}=500 \mathrm{~mm}\right)$ was chosen so entire image is seen. In the presented view in Fig. 8(a) two gaps are seen. The size of the viewed gap is $\mathrm{MFoV}_{\text {gap }}=6.5 \mathrm{~mm}$. The size of viewed gap in presented images and videos appears to be smaller. In some area of the gap we see the image of decreased intensity and resolution. This area can be computed from WD chart in Fig. 4(b).

The image and accompanying videos presented in Fig. 8(a) are captured while observing an image through the asymmetric diffuser. The asymmetric diffuser is placed in the center of the real image reconstruction space as shown in Fig. 4. The diffuser scatters light approximately in one direction only (y). Scattering angles of applied diffuser are $0.2^{\circ} \times 40^{\circ}$. This extends MFOV in the y direction and allows for a more convenient holographic image observation i.e., the eye may take a wide range of positions in the $y$-plane. We note that the perspective of the holographic image in the $x$-direction is unaffected. In Fig. 8(a), three stripes are seen, they are representing views given at asymmetric diffuser by separate SLMs. The width of the single stripe corresponds to MFOV in $x$-direction. 


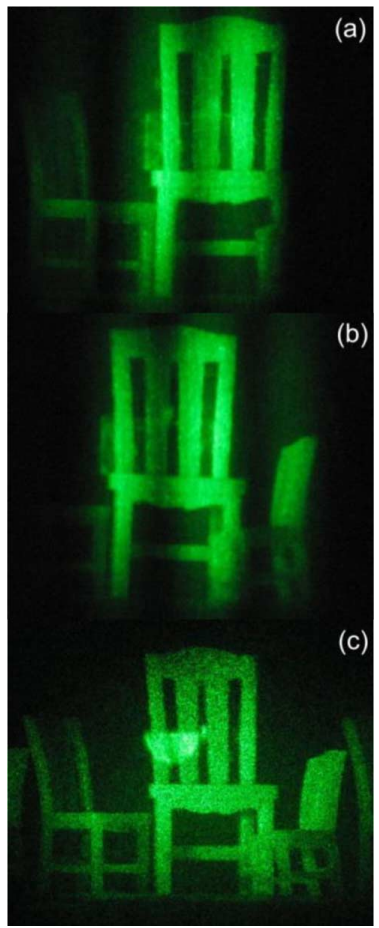

Fig. 9. Optical reconstructions of the chairs scene captured with digital holography, view captured with the asymmetric diffuser and digital camera (Media 3). (a) Left perspective; (b) right perspective; and (c) views captured with the eyepiece lens and digital camera (Media 4).

In Fig. 8(b) we show the view (image and video) of the same holographic scene, however instead of the diffuser we now place an eyepiece lens of focal length $200 \mathrm{~mm}$ generating a virtual image of the object. With eyepiece the observation is not as convenient as with an asymmetric diffuser. The eye cannot take a wide range of positions, it has to be positioned in the location of one of real images of SLMs given by eyepiece. Both videos are captured with a digital camera moving on a linear stage so that different perspectives of the reconstruction space can be seen. During capturing Media 1 the camera was moved in the range: $x_{o} \in(-60 \mathrm{~mm}, 60 \mathrm{~mm})$.

In our digital holography setup we have captured another scene. The scene is composed from three small chairs of different sizes. The scene elements were printed using a 3D printer (ZPrinter 650). The results of the reconstructions are presented in Fig. 9. Once again the scene was captured for $\mathrm{FF}=0.51\left(\Delta_{\alpha 2}=2.30^{\circ}\right)$ and the reconstruction distance $z_{2}=750 \mathrm{~mm}$. All other parameters are the same as in the previous experiment. The scene size in the $x$ direction was chosen to be close to the $\mathrm{FoV}(\mathrm{FoV}=49.9 \mathrm{~mm})$. For this scene size the reconstructed images of the zero and first diffraction orders are next to each other. Presented views were obtained for the observation distance $z_{o}=600 \mathrm{~mm}$, where the $\mathrm{MFoV}_{\mathrm{FF}}=33.6$ $\mathrm{mm}\left(M F o V_{\mathrm{FF}}<\mathrm{FoV}\right)$. In this case we cannot view the entire scene with an eye. In Fig. 9 we present two perspectives captured with asymmetric diffuser; (a) left one and the right one (b). The video (Media 3) was taken with digital camera moving in range $x_{o} \in(-75 \mathrm{~mm}, 75 \mathrm{~mm})$. Fig. 9(c) shows the central perspective obtained with the eyepiece.

\section{CONCLUSION}

In this paper, we present the concept of recording and displaying real world 3D objects using multiple digital holograms arranged in a circular configuration. We derive theoretical fundamentals governing circular arrangements of: 1) CCDs for digital hologram capture and 2) phase SLMs for their display. In our experiments however, while we do employ a circular configuration of SLMs for display, we use only a single camera for capture where we rotate the object between captures in order to simulate a multi-camera set up. Nevertheless we believe that our theoretical and experimental results are general to the multi-camera recording arrangement. In our theoretical investigation we consider: 1) the analysis of information content of the wide viewing angle holographic capture and display systems; 2) the influence of the capture, display configurations mismatch on the 3D imaging properties; and 3) visual perception analysis of the novel display. We give special attention to the problem of gaps between CCDs during object wavefront capture and gaps between the active areas of the SLMs during the 3D image reconstruction process. Based on a Wigner distribution function analysis we derive formulae for a number of important properties. We show the lack of information about an object within specific angular field of views determined by the presence of gaps in the captured or/and reconstructed signals and this is demonstrated by experiment. Both theoretically and experimentally we show the effect of gaps on human-visual perception.

In our experiments, using a single camera and a rotation stage, it is also shown that in the case of a static real world object we are able to record a scene with unitary fill factor. However this is difficult to achieve in the case of a time varying object (scene) for which the multi-camera capture system shown in Fig. 1(a) should be used. For our multi-SLM display system the fill factor is less than unity as the SLM frame (beam splitting cubes) determines the distance between neighboring active SLM areas. It can be increased if we use a display configuration that is decoupled from the capture system, but such a system may require the processing of the captured holograms for the display geometry [21]. The requirements which allow us to reduce the mismatch between the capture and display systems are discussed and it is shown that having the knowledge of the basic parameters of both systems this mismatch can be removed fully or partially.

It is important to mention that in our display we are unable to coherently stitch together the individual wave fields from two SLMs in order to create a true synthetic aperture wavefield. To do this accurately we would have to position the SLMs relative to one another with an accuracy in the order of a fraction of a wavelength, and in turn we would have to measure our relative CCD positions with similar accuracy.

\section{REFERENCES}

[1] B. Javidi and F. Okano, Three Dimensional Television, Video and Display Technologies. Berlin, Germany: Springer, 2002.

[2] H. M. Ozaktas and L. Onural, Three-Dimensional Television. Berlin, Germany: Springer, 2008.

[3] , B. Javidi, F. Okano, and J.-Y. Son, Eds., Three-Dimensional Imaging, Visualization and Display. Berlin, Germany: Springer, 2009

[4] E. N. Leith, J. Upatnieks, B. P. Hildenbrand, and K. Haines, "Requirements for the wavefront reconstruction television facsimile system," $J$. SMPTE, vol. 74, pp. 893-896, 1965. 
[5] Digital Holography and Three-Dimensional Display, T.-Ch. Poon, Ed. Berlin, Germany: Springer, 2006.

[6] P. S. Hilarie, S. A. Benton, M. Lucente, and P. M. Hubel, "Color images with the MIT holographic video display," in Proc. SPIE, 1992, vol. 1667 , pp. 73-84.

[7] R. Haussler, A. Schwerdtner, and N. Leister, "Large holographic display as an alternattive to stereoscopic displays," in Proc. SPIE, 2008, vol. 6803 , p. $68030 \mathrm{M}$.

[8] M. Stanley et al., "3D electronic holography system using a 100 mega-pixel spatial light modulator," in Proc. SPIE, 2004, vol. 5249, pp. 297-308.

[9] F. Yaras, H. Kang, and L. Onural, "Multi-SLM holographic display system with planar configuration," IEEE, 2010.

[10] J. Hahn, H. Kim, Y. Lim, G. Park, and B. Lee, "Wide viewing angle dynamic holographic stereogram with a curved array of spatial light modulators," Opt. Express, vol. 16, pp. 12372-12386, 2008.

[11] F. Yaraş, H. Kang, and L. Onural, "Circular holographic video display system," Opt. Express, vol. 19, pp. 9147-9156, 2011.

[12] G. Finke, T. Kozacki, and M. Kujawinska, "Wide viewing angle holographic display with multi spatial light modulator array," in Proc. SPIE, 2010, vol. 7723, p. 77230A.

[13] M. Kujawinska, T. Kozacki, D. P. Kelly, and D. S. Monaghan, "Numerical conversion and optoelectronic reconstruction of digital holograms by multiple spatial light modulators," in Proc. SPIE, 2010, vol. 7790, p. $77900 \mathrm{R}-25$.

[14] A. W. Lohmann, R. G. Dorsch, D. Mendlovic, Z. Zalevsky, and C. Ferreira, "Space-bandwidth product of optical signals and systems," $J$. Opt. Soc. Amer. A, vol. 13, pp. 470-473, 1996.

[15] M. Testorf, B. M. Hennelly, and J. O. Castenada, Phase Space Optics. New York: McGraw-Hill, 2009.

[16] S. Sinzinger and J. Jahns, Microoptics, 2nd ed. : Wiley-VCH, 2003.

[17] D. P. Kelly et al., "Digital holographic capture and optoelectronic reconstruction for 3D displays," Int. J. Digital Multimedia Broadcast., 2010.

[18] J. W. Goodman, Introduction to Fourier Optics, 2nd ed. New York: McGraw-Hill, 1996.

[19] T. Kozacki, "On resolution and viewing of holographic image generated by 3D holographic display," Opt. Express, vol. 18, pp. 27118-27129, 2010.

[20] I. Yamaguchi and T. Zhang, "Phase-shifting digital holography," Opt. Lett., vol. 22, pp. 1268-1270, 1997.

[21] T. Kozacki, "Holographic display with tilted spatial light modulator," Appl. Opt., vol. 50, pp. 3579-3588, 2011.
Tomasz Kozacki received the Ph.D. degree in photonics from the Warsaw University of Technology, Warsaw, Poland, in 2005.

$\mathrm{He}$ is currently works as an assistant professor with the Warsaw University of Technology, Warsaw, Poland. His scientific research are related to digital holography, holographic displays, holographic microscopy, computational diffraction and optical diffraction tomography. He has authored and co-authored 12 scientific journal publications, and more than 20 conference proceedings.

Małgorzata Kujawinska has received the Ph.D. and D.Sc. degrees from Warsaw University of Technology in 1990.

She is a Full Professor of applied optics at Warsaw University of Technology, Head of Photonics Engineering Division at Institute of Micromechanics and Photonics. She is an expert in full-field optical metrology, image processing, automatic data analysis and design of novel photonics systems. Author of one monograph, several book chapters and more than 200 papers in international scientific journals.

Dr. Kujawinska is a Fellow of SPIE.

Grzegorz Finke received the M.Sc. degree from Warsaw University of Technology, Warsaw, Poland, in 2008, where he is currently working toward the $\mathrm{Ph} . \mathrm{D}$. degree in the field of digital holography, and is a participant of $3 D$ Real EU project.

Mr. Finke is a member of SPIE.

Weronika Zaperty is currently working toward the M.Sc. degree from Warsaw University of Technology, and is a participant of 3 DReal EU project.

Mr. Zaperty is a member of SPIE.

Bryan Hennelly received the Ph.D. dgeree in optical physics from the University College Dublin in Ireland in 2004. He is currently a research fellow at the National University of Ireland Maynooth. His research interests include digital holography, Wigner Distribution functions, simulation of optical systems and Raman spectroscopy and is the author or co-author of numerous articles in these topics. 